\title{
Estrutura Fractal em Mercados Emergentes
}

\author{
Tulio Silva Ribeiro \\ Ricardo Pereira Câmara Leal
}

\section{Resumo}

A Hipótese de Eficiência do Mercado é uma das bases da moderna teoria de finanças e estabelece que o comportamento aleatório na variação dos preços decorre do fluxo de informações não antecipadas. Um de seus paradigmas define que a distribuição dos retornos dos preços é aleatória e normalmente distribuída. Neste artigo, avaliamos a hipótese de que o processo estocástico gerador dos retornos de diversos mercados emergentes da Ásia e das Américas segue um processo aleatório não-normal alfa-estável. Por meio de estimativas dos parâmetros da distribuição e de simulações, encontramos evidências de que esses retornos realmente seriam mais bem descritos pela distribuição alfa-estável ou distribuição fractal. Estas distribuições acomodam flutuações grandes e freqüentes de preço melhor, pois a probabilidade de perdas substanciais é maior do que a prevista por uma distribuição normal e a assimetria da distribuição é considerada. As estimativas fornecidas para modelos usuais de finanças, como os otimizadores de média e variância de Markowitz e o modelo de apreçamento de opções de Black e Scholes, podem ser mais bem estimadas por meio da distribuição alfa-estável.

Palavras-chaves: fractais; eficiência de mercado; distribuições alfa-estáveis; valores extremos; mercados emergentes.

\section{Abstract}

The Efficient Markets Hypothesis is one of the fundamental concepts of modern financial theory. It states that the random behavior of prices stems from the unpredictable information flow. One of its paradigms defines the random returns distribution as normal. In this article we evaluate the hypothesis that the stochastic return generating process in the emerging markets of Asia and Latin America follows a non-normal alpha stable process. We estimate the distribution parameters through maximum likelihood estimation and simulation and present evidence that stock market returns would be better described by the alpha stable or fractal distribution. These distributions accommodate large and frequent price fluctuations as well as asymmetry, when the likelihood of large losses is greater than what would be warranted under a normal distribution. Inputs to usual financial models, such as Markowitz' mean-variance optimizers and the Black and Scholes option pricing model, may be better estimated by means of an alpha stable distribution.

Key words: fractals; efficient market hypothesis; alpha-stable distributions; extreme values; emerging markets. 


\section{INTRODUÇÃO}

A Teoria de Eficiência do Mercado é uma das bases da moderna teoria de finanças. Segundo ela, o comportamento aparentemente aleatório na variação dos preços decorre do fluxo randômico de informações não antecipadas que os afetam. Uma de suas formulações usuais define que a distribuição das variações dos preços de mercado, além de aleatória, é normalmente distribuída. Torres, Bonomo e Fernandes (2000) rejeitam tanto a linearidade quanto a normalidade dos retornos para o mercado brasileiro ${ }^{(1)}$.

O processo estocástico dos retornos é objeto de estudos há décadas. Bachelier (1900) desenvolveu o modelo pioneiro no qual as variações nos preços de um ativo eram variáveis aleatórias independentes e identicamente distribuídas (i.i.d.), com variância finita e, de acordo com o Teorema do Limite Central, essas variações poderiam ser descritas por uma distribuição normal. Posteriormente, Osborne (1959) refinou esses resultados, de modo que os retornos foram caracterizados por uma distribuição log-normal.

$\log \left[P(t+d t)-\log [P(t)] \sim N\left(\mu d t, \sigma^{2} d t\right)\right.$

Diversos estudos questionaram este modelo e verificou-se que os retornos apresentavam distribuições assimétricas e leptocúrticas incompatíveis com uma distribuição gaussiana que também subestima a ocorrência de observações extremas. Mandelbrot (1963) observou que a distribuição dos retornos seria mais bem descrita por uma distribuição alfa-estável. Esta classe de distribuições, desenvolvida por Lévy (1925), é uma generalização do Teorema do Limite Central para a soma de variáveis aleatórias i.i.d., sem restrições em relação ao segundo momento. Um de seus casos especiais é a distribuição de Gauss. Subseqüentemente, diversos estudos confirmaram a hipótese alfa-estável em diversos mercados ${ }^{(2)}$.

A hipótese de que um mercado segue uma distribuição alfa-estável tem diversas conseqüências. Estas distribuições acomodam grandes flutuações que ocorrem com maior freqüência, assim como mais volatilidade, que levam à probabilidade de perdas substanciais. As conseqüências práticas para o uso destas distribuições seriam estimativas melhores dos parâmetros utilizados em modelos financeiros como o CAPM, a otimização de média e variância de Markowitz ou o modelo de avaliação de opções de Black e Sholes. Um dos principais problemas que temos ao usar um otimizador de Markowitz, por exemplo, é a matriz de covariância a 
ser fornecida. O problema de erro nos dados é grave, quando se usam estimativas históricas não condicionais, levando vários autores a sugerirem formas alternativas de se estimar a fronteira eficiente, como a derivação de uma fronteira eficiente estatística por meio de técnicas de simulação de bootstrap (Michaud, 1998; Leal, Da Silva e Ribeiro, 2001).

Essas observações são ainda mais pertinentes, quando relacionadas a mercados emergentes. O IFC (1999) estabelece que os mercados de capitais de países em desenvolvimento são emergentes. Esses mercados ainda estão num processo de integração ao cenário global e suas economias possuem significativas diferenças em relação às de países desenvolvidos, sofrendo, nas duas últimas décadas, diversas reformas políticas e estruturais (Bekaert, 1993). Além disso, elas são mais voláteis e as crises recentes, como as de 1997-1999, têm mostrado que há riscos substanciais. A distribuição empírica dos retornos reflete tais fatos.

Neste artigo, avaliamos a hipótese de que os retornos dos mercados emergentes sejam descritos melhor por uma distribuição alfa-estável com uma amostra que cobre um período de aproximadamente 18 anos para alguns dos maiores mercados emergentes da Ásia e da América Latina, além de Hong Cong, Cingapura, Alemanha, Japão, Inglaterra e Estados Unidos. A fim de comparar os resultados com o comportamento de carteiras bem diversificadas de ativos, tanto mundiais como regionais, investigamos o comportamento dos índices da Morgan Stanley Capital International (MSCI) para o mundo (World Index), oriente (Far East Index), América Latina e para o conjunto dos mercados emergentes.

\section{Metodologia e Dados}

\section{Distribuições Alfa-Estáveis}

Segundo o Teorema do Limite Central, a soma parcial $P_{n} \equiv \sum_{i=1}^{n} x_{i}$ para variáveis aleatórias $x_{i}$ i.i.d., no caso dos $x_{i}$ 's possuírem segundo momento finito, teria uma distribuição de Gauss no limite $n \rightarrow \infty$. Lévy (1925) generalizou o Teorema do Limite Central, não impondo nenhuma restrição ao segundo momento. Nesse caso, o único limite não-trivial possível para $P_{n}$ é uma distribuição alfaestável. Exceto para casos especiais, como as distribuições de Gauss e de Cauchy, as distribuições estáveis não possuem fórmulas fechadas. Geralmente são expressas pela transformação de Fourier ou funções características.

Existem diversas parametrizações para distribuições alfa-estáveis. A mais usual atualmente (ver Samorodnitsky e Taqqu, 1994) é a seguinte: 


$$
\begin{aligned}
& X \sim f_{1}(\alpha, \beta, \gamma, \delta) \Leftrightarrow \\
& E \exp (i t X)=\left\{\begin{array}{ll}
\exp \left\{-\gamma^{\alpha}|t|^{\alpha}\left[1-i \beta\left(\tan \frac{\pi \alpha}{2}\right)(\operatorname{sign}(t))\right]+i \delta t\right\} & \alpha \neq 1 \\
\exp \left\{-\gamma|t|\left[1-i \beta \frac{\pi}{2}(\operatorname{sign}(t)) \ln |t|\right]+i \delta t\right\} & \alpha=1
\end{array}\right\}
\end{aligned}
$$

$\alpha \in(0,2], \beta \in[-1,1], \gamma \geq 0$ e $\delta \in R$, onde $R \quad$ é o conjunto dos números reais.

As distribuições estáveis são caracterizadas pelos quatro parâmetros $\alpha, \beta, \gamma, \delta ; \gamma$ é o parâmetro de escala e $\delta$ o de locação. O parâmetro de locação corresponde à expectativa da média para $\alpha>1$. Quando $\alpha=2$, o desvio padrão da distribuição corresponde a $\gamma / \sqrt{2}$.

Os parâmetros $\alpha$ e $\beta$ são relativamente mais importantes e determinam a forma da distribuição; $\beta$ é o parâmetro de assimetria e varia entre -1 e 1 . Quando tem o valor 0 , a distribuição é simétrica; $\alpha$ é o parâmetro de estabilidade ou expoente característico e varia entre 0 e $2 ; \alpha=2$ corresponde a uma distribuição gaussiana. Esse parâmetro determina a altura das caudas e a curtose em $\delta$. Quanto menor for $\alpha$, mais gordas são as caudas. Uma distribuição tem caudas gordas, se elas forem mais pesadas que a exponencial. Para $\alpha<2$, as distribuições alfa-estáveis têm uma cauda (quando $\alpha<1$ e $\beta= \pm 1$ ) ou ambas as caudas que são assintoticamente power laws com caudas gordas. Como consequiência, variância finita existe somente para $\alpha=2$ e média finita somente para $\alpha>1$.

Por esses motivos, muitos são levados a rejeitar a hipótese de uma distribuição alfa-estável não gaussiana. Nesse caso, vale distinguir entre variância da população e da amostra.

i. Variância da População:

$$
\sigma^{2}=E(X-\mu)^{2}
$$

ii. Variância da Amostra:

$$
s_{n}^{2}=\frac{\sum_{i=1}^{n}\left(X_{i}-\bar{X}\right)^{2}}{n-1}
$$


A primeira é uma integral em intervalo infinito, que é finita somente se as caudas da distribuição não forem gordas, enquanto (ii) é uma soma finita, portanto, sempre é possível calcular a posteriori a variância de uma amostra. Mas, com o aumento da amostra, verifica-se que essa variância não converge (Peters, 1994). A variância é uma das medidas de dispersão e não é apropriada para todos os problemas. Os mesmos comentários são pertinentes em relação à média.

Entre as diversas propriedades dessa classe de distribuições, duas merecem destaque. Na distribuição de Gauss, a soma de duas variáveis aleatórias normais é uma variável aleatória normal. Assim, se $X$ é normal, para $X_{1}$ e $X_{2}$ cópias independentes de $X$ e qualquer constante positiva $a$ e $b$,

$a X_{1}+b X_{2} \stackrel{d}{=} c X+d$

que vale para distribuições alfa-estáveis, desde que possuam o mesmo $\alpha$ e $\beta$. A outra propriedade é a de auto-similaridade. Assim, $\alpha$, $\beta$ e $\delta$ devem ser constantes em diferentes escalas, ou seja, não mudariam independentemente da freqüência dos dados. Essa característica levou esta classe de distribuições a ser referida também como distribuições fractais.

\section{Dados}

Nossos dados consistem no valor de fechamento, em dólares americanos, para alguns dos maiores mercados emergentes segundo classificação do IFC. Analisamos os índices Hang Seng (Hong Kong), Singapore Straits Industrial (Cingapura), SIBI General (Argentina), IBOVESPA (Brasil), IGPA (Chile), Bombay SE 30 (Índia), Korea SE Composite Index (Coréia do Sul), Kuala Lumpur Composite Index (Malásia), IPYC (México), Manila Composite Index (Filipinas), Taiwan SE Weighted Index (Taiwan), Bangkok SET Index (Tailândia), Johannesburg SE All Share (África do Sul) e Jakarta Composite (Indonésia). Incluímos na análise, também, os indices Standard \& Poor’s 500 (Estados Unidos), Nikkei 225 (Japão), FTSE All Shares (Inglaterra), DAX 30 (Alemanha) e os índices regionais da Morgan Stanley: World Index, Far East Index, Latin American Index e Emerging Markets Index. Os indices citados não incluem dividendos e foram coletados na base de dados Datastream.

Os períodos analisados variam devido à dificuldade de se conseguir séries longas para mercados emergentes. A maior amostra foi a do IBOVESPA brasileiro, cobrindo de 05/02/1982 a 31/12/1999. A menor amostra, do Jakarta Composite da Indonésia, de 29/12/1989 a 31/12/1999. Eliminamos os valores correspondentes a dias de não negociação.

Para a análise, transformamos os índices diários em taxas diárias de retorno na 
forma logarítmica. Dividimos cada amostra em diversas amostras com 500 observações, aproximadamente 2 anos, de dias de negociação consecutivos. Essa separação foi feita do fim para o começo, de modo que, para todos os índices, terminasse no mesmo dia. A porção da amostra referente ao início do período inevitavelmente teria menos de 500 observações e foi eliminada. Os períodos utilizados são ligeiramente diferentes para cada país, uma vez que todos têm 500 dias de negócio e foram contados de 30/ 12/1999 para trás. A subdivisão da amostra resultou em até 9 subperíodos. Para haver consistência na análise, forçamos a data de início do primeiro subperíodo de cada país para que fosse a mesma que a usada para a amostra empregada na análise do período total, mesmo que o país dispusesse de informações anteriores a esta data.

\section{Metodologia}

Antes de estimarmos os parâmetros para as amostras, verificamos se todas eram unimodais. Caso existissem múltiplas modas, então não poderia haver um processo alfa-estável. Fizemos, portanto, gráficos de densidade suavizados para todas as amostras totais e parciais. Como observado em Nolan (1999), “a largura do kernel geralmente sugerida de $2 n^{-1 / 3}$, funcionava razoavelmente, quando as caudas não eram muito pesadas, algo como $\alpha>1,5$, mas não funcionava bem para caudas mais pesadas". Nesses casos procuramos utilizar a menor largura do kernel possível, suficiente para não mostrar oscilações de pontos individuais. Não verificamos múltiplas modas em nenhumas das amostras. Os gráficos foram omitidos para poupar espaço.

Para estimar os parâmetros das distribuições, utilizamos o método de máxima verossimilhança, inicialmente proposto por Dumochel (1971), implementado no programa STABLE, desenvolvido por John P. Nolan. Este método, entre outras vantagens, oferece-nos intervalos de confiança. Utilizamos, também, outra parametrização para a função característica, uma variação da parametrização (M) de Zolotarev (1986):

$$
\begin{aligned}
& X \sim f_{0}(\alpha, \beta, \gamma, \delta) \Leftrightarrow \\
& E \exp (i t X)=\left\{\begin{array}{lc}
\exp \left\{-\gamma^{\alpha}|t|^{\alpha}\left[1+i \beta\left(\tan \frac{\pi \alpha}{2}\right)(\operatorname{sign}(t))\left(\left(\gamma^{\alpha}|t|^{1-\alpha}\right)-1\right)\right]+i \delta t\right\} \quad \alpha \neq 1 \\
\exp \left\{-\gamma|t|\left[1+i \beta \frac{\pi}{2}(\operatorname{sign}(t))(\ln |t|+\ln \gamma)\right]+i \delta t\right\} & \alpha=1
\end{array}\right\}
\end{aligned}
$$

$\alpha \in(0,2], \beta \in[-1,1], \gamma \geq 0$ e $\delta \in R$, onde R é o conjunto dos números reais. 
A vantagem do uso dessa representação é que a função característica - e as correspondentes densidades e funções de distribuição - são conjuntamente contínuas nos quatro parâmetros, o que não pode ser verificado na outra parametrização $f_{1}(\alpha, \beta, y, \delta)$ descrita na seção anterior. Além disso, $\alpha$ e $\beta$ possuem um significado mais claro como medidas de peso das caudas e de assimetria (Nolan, 1999).

Verificamos, então, o ajuste do método de máxima verossimilhança. Utilizamos o pp-plot proposto em Michael (1983), pois o convencional tende a enfatizar o comportamento da distribuição na moda, distorcendo a curva nas caudas. Este pp-plot apresenta melhores resultados na verificação dos ajustes nas caudas. Em seguida, comparamos os resultados com as estimativas pelo método de quantis (McCulloch, 1986). Caso diferissem signicativamente, haveria indícios de que não seria uma distribuição alfa-estável. Por fim, utilizamos os parâmetros estimados nos períodos parciais de cada série para gerar valores aleatórios com amostras de mesmo tamanho $(n=500)$. Reunimos as amostras geradas em uma amostra total e reestimamos os parâmetros da distribuição resultante.

\section{Resultados e Conclusões}

A Tabela 1 apresenta as estimativas para $\alpha$ e $\beta$ para os mercados selecionados, comparando as estimativas obtidas por meio dos diferentes métodos. Em nenhum caso encontramos diferenças que justificassem rejeitar a hipótese alfa-estável. Os índices relativos a países desenvolvidos obtiveram os maiores parâmetros de estabilidade, mas ainda assim com uma diferença significativa para a hipótese gaussiana de $\alpha=2$, pois não há intervalo de confiança, seja por que método for, que inclua este valor. No caso de mercados emergentes, essa diferença é ainda mais notável. Todos apresentaram $\alpha>1,65$, sendo o menor referente à Indonésia, igual a 1,09. Lembramos que quanto menor for $\alpha$, mais gordas são as caudas. Portanto o resultado encontrado indica que a suposição de um processo estocástico com distribuição normal não é apropriada e que estimativas a partir de dados históricos devem considerar que as caudas são gordas e com probabilidade maior do que a da curva normal de ocorrência de valores extremos. Os mercados emergentes tendem a apresentar valores de alfa menores do que os observados para os mercados desenvolvidos mas nossas recomendações valem para todos os mercados. Lembramos que variância finita existe somente para $\alpha=2$ e média finita, somente para $\alpha>1$. Portanto métodos de estimação robustos devem ser usados quando se precisar obter retornos esperados, matrizes de covariância ou uma volatilidade implícita, pois todas as distribuições empíricas podem ser descritas 
por uma distribuição alfa-estável, cujo alfa é significativamente menor do que 2. Quando beta é nulo, a distribuição é simétrica. Os betas na Tabela 1 indicam que não se pode rejeitar a hipótese de uma distribuição simétrica para a maioria dos países. No caso do Brasil, parece que os eventos extremos negativos são mais freqüentes do que seria de se esperar para uma distribuição alfa-estável.

\section{Tabela 1: Estimativas Obtidas por Máxima Verossimilhança para $\alpha$ e $\beta$ de uma Distribuição Alfa-Estável para Mercados Selecionados, Comparadas às Estimativas Obtidas pelo Método de Quantis e pela Amostra Simulada}

\begin{tabular}{|l|r|r|r|r|r|r|r|r|r|r|}
\hline & \multicolumn{9}{|c|}{ Máxima Verossimilhança } & \multicolumn{2}{|c|}{ Quantis } & \multicolumn{3}{|c|}{ Simulação } \\
\hline Países & alfa & IC Inf. & IC Sup. & Beta & IC $(+/$-) & Alfa & Beta & Alfa & IC Inf. & IC Sup. \\
\hline Inglaterra & 1.734 & 1.691 & 1.777 & $\mathbf{- 0 . 1 5 5}$ & 0.136 & 1.733 & -0.155 & 1.746 & 1.703 & 1.789 \\
\hline Alemanha & 1.665 & 1.620 & 1.710 & -0.061 & 0.117 & 1.665 & -0.061 & 1.699 & 1.654 & 1.744 \\
\hline MSCI World & 1.604 & 1.558 & 1.651 & 0.004 & 0.105 & 1.604 & -0.001 & 1.649 & 1.603 & 1.695 \\
\hline África do Sul & 1.544 & 1.491 & 1.596 & 0.004 & 0.109 & 1.544 & 0.004 & 1.563 & 1.511 & 1.616 \\
\hline MSCI L.America & 1.541 & 1.485 & 1.598 & 0.008 & 0.112 & 1.541 & -0.184 & 1.560 & 1.503 & 1.616 \\
\hline Estados Unidos & 1.531 & 1.484 & 1.577 & 0.006 & 0.094 & 1.531 & 0.006 & 1.611 & 1.565 & 1.658 \\
\hline Brasil & 1.516 & 1.469 & 1.562 & $\mathbf{- 0 . 1 1 5}$ & 0.089 & 1.516 & -0.115 & 1.502 & 1.456 & 1.548 \\
\hline México & 1.506 & 1.460 & 1.552 & -0.073 & 0.089 & 1.468 & -0.090 & 1.429 & 1.382 & 1.475 \\
\hline Cingapura & 1.501 & 1.449 & 1.554 & -0.042 & 0.101 & 1.501 & -0.042 & 1.441 & 1.388 & 1.494 \\
\hline MSCI Emerging & 1.500 & 1.444 & 1.557 & $\mathbf{- 0 . 2 0 3}$ & 0.104 & 1.500 & -0.203 & 1.509 & 1.453 & 1.566 \\
\hline India & 1.495 & 1.446 & 1.544 & $\mathbf{0 . 1 1 7}$ & 0.092 & 1.495 & 0.117 & 1.520 & 1.471 & 1.569 \\
\hline MSCI Far East & 1.476 & 1.427 & 1.525 & 0.042 & 0.091 & 1.476 & 0.042 & 1.570 & 1.520 & 1.619 \\
\hline Hong Kong & 1.465 & 1.412 & 1.517 & -0.035 & 0.096 & 1.465 & -0.035 & 1.493 & 1.440 & 1.545 \\
\hline Japão & 1.460 & 1.414 & 1.506 & -0.043 & 0.084 & 1.460 & -0.043 & 1.486 & 1.440 & 1.532 \\
\hline Coréia do Sul & 1.414 & 1.365 & 1.462 & 0.055 & 0.084 & 1.414 & 0.055 & 1.423 & 1.374 & 1.472 \\
\hline Malásia & 1.393 & 1.350 & 1.441 & -0.017 & 0.078 & 1.395 & -0.017 & 1.451 & 1.405 & 1.496 \\
\hline Taiwan & 1.393 & 1.344 & 1.441 & -0.059 & 0.081 & 1.393 & -0.059 & 1.376 & 1.327 & 1.424 \\
\hline Chile & 1.382 & 1.336 & 1.428 & 0.029 & 0.076 & 1.382 & 0.029 & 1.369 & 1.323 & 1.414 \\
\hline Filipinas & 1.365 & 1.317 & 1.413 & 0.028 & 0.079 & 1.365 & 0.028 & 1.419 & 1.371 & 1.468 \\
\hline Argentina & 1.363 & 1.315 & 1.412 & 0.020 & 0.079 & 1.363 & 0.020 & 1.381 & 1.333 & 1.429 \\
\hline Tailândia & 1.278 & 1.231 & 1.325 & -0.046 & 0.071 & 1.278 & -0.046 & 1.321 & 1.275 & 1.368 \\
\hline Indonésia & 1.090 & 1.036 & 1.144 & -0.038 & 0.075 & 1.090 & -0.038 & 1.087 & 1.033 & 1.141 \\
\hline
\end{tabular}

Nota: a tabela está em ordem crescente de $\alpha$ estimado por máxima verossimilhança. Os valores em negrito são significativamente diferentes do que se esperaria para uma distribuição alfa-estável.

Como os parâmetros flutuam no tempo, realizamos simulações com a amostra total. A Tabela 1 mostra uma comparação entre o $\alpha$ estimado a partir dos dados empíricos e o alfa estimado a partir da amostra cujos valores foram gerados de acordo com os parâmetros das amostras parciais. Os resultados mostram que o processo estocástico dos retornos na amostra total é bem caracterizado por um processo alfa-estável apesar dos parâmetros variarem no tempo.

A Tabela 2 apresenta as estimativas para o alfa em cada subperíodo. Vale ressaltar o comportamento do expoente característico em face das crises globais. Nos períodos de crises percebe-se que o $\alpha$ foi significativamente mais baixo em diversos mercados, resultado das freqüentes e grandes flutuações. No período 8, 
quando ocorreu a crise da Ásia, a maioria dos países asiáticos e todos os índices MSCI apresentaram o alfa mais baixo. Nos demais mercados, o alfa baixo corresponde a períodos de crise local. Aggarwal, Leal e Inclán (1999) já haviam notado que os períodos de mudança de regime na volatilidade dos mercados emergentes coincidem com crises locais, sejam políticas, econômicas ou sociais. Portanto o processo estocástico dos retornos dos mercados analisados é mais bem caracterizado por uma distribuição alfa estável, particularmente em períodos de crise. Isto implica dizer que uma média aritmética simples e o desvio padrão dos retornos podem não ser boas estimativas do retorno esperado e do risco na presença de valores extremos mais freqüentes do que quando a distribuição da população é normal. O paradigma da normalidade, quando se calculam estimativas para usar em otimização de média e variância ou quando se usa o modelo de Black e Scholes, por exemplo, pode estar subestimando os riscos.

Tabela 2: Estimativas para o $\alpha$ em cada Subperíodo

\begin{tabular}{|l|r|r|r|r|r|r|r|r|r|}
\cline { 2 - 10 } \multicolumn{1}{c|}{} & \multicolumn{9}{|c|}{ Período } \\
\cline { 2 - 10 } \multicolumn{1}{c|}{} & 1 & 2 & 3 & 4 & 5 & 6 & 7 & 8 & 9 \\
\hline Alemanha & 2.00 & 1.80 & 1.57 & 1.87 & 1.61 & 1.88 & 1.90 & 1.61 & $\mathbf{1 . 5 6}$ \\
\hline Argentina & & 1.67 & 1.59 & 1.46 & 1.57 & 1.73 & 1.49 & 1.72 & $\mathbf{1 . 4 1}$ \\
\hline Brasil & 1.59 & 1.61 & 1.89 & 1.85 & 1.81 & 1.84 & 1.58 & $\mathbf{1 . 4 5}$ & 1.57 \\
\hline Chile & 1.19 & 1.33 & 1.62 & 1.45 & $\mathbf{1 . 0 3}$ & 1.30 & 1.65 & 1.94 & 1.53 \\
\hline Cingapura & & & 1.68 & 1.62 & 1.54 & 1.61 & 1.65 & $\mathbf{1 . 4 4}$ & 1.63 \\
\hline Coréia do Sul & & 1.53 & 1.68 & 1.85 & $\mathbf{1 . 3 1}$ & 1.69 & 1.65 & 1.38 & 1.59 \\
\hline Estados Unidos & 1.67 & 1.78 & $\mathbf{1 . 3 6}$ & 1.50 & 1.68 & 1.54 & 1.61 & 1.60 & 1.86 \\
\hline Filipinas & & $\mathbf{1 . 1 4}$ & 1.32 & 1.50 & 1.58 & 1.68 & 1.75 & 1.37 & 1.44 \\
\hline Hong Kong & & & 1.61 & 1.56 & 1.77 & 1.54 & 1.68 & $\mathbf{1 . 3 5}$ & 1.60 \\
\hline Índia & & $\mathbf{1 . 3 3}$ & 1.44 & 1.70 & 1.52 & 1.62 & 1.70 & 1.61 & 1.64 \\
\hline Indonésia & & & & & 1.36 & 1.43 & 1.49 & $\mathbf{1 . 1 0}$ & 1.42 \\
\hline Japão & 1.84 & 1.54 & 1.65 & 1.59 & 1.54 & 1.44 & 1.48 & $\mathbf{1 . 4 3}$ & 1.72 \\
\hline Malásia & 1.75 & 1.46 & 1.62 & 1.34 & 1.40 & 1.47 & 1.66 & $\mathbf{1 . 2 2}$ & 1.47 \\
\hline México & $\mathbf{1 . 1 9}$ & 1.57 & 1.41 & 1.38 & 1.74 & 1.66 & 1.38 & 1.66 & 1.72 \\
\hline Tailândia & & 1.50 & 1.36 & 1.56 & 1.46 & 1.59 & 1.62 & $\mathbf{1 . 3 0}$ & 1.51 \\
\hline Taiwan & & 1.65 & 1.43 & 1.86 & 2.00 & $\mathbf{1 . 4 2}$ & 1.55 & 1.44 & 1.49 \\
\hline MSCI Emerging & & & & 1.87 & $\mathbf{1 . 4 4}$ & 1.67 & 1.55 & $\mathbf{1 . 4 4}$ & 1.73 \\
\hline MSCI Far East & & 1.67 & 1.61 & 1.49 & 1.64 & 1.64 & 1.84 & $\mathbf{1 . 4 2}$ & 1.67 \\
\hline MSCI Latin America & & & & 1.61 & 1.73 & 1.87 & 1.52 & $\mathbf{1 . 3 9}$ & 1.57 \\
\hline MSCI World & 1.74 & 1.95 & 1.61 & 1.81 & 1.55 & 1.70 & 1.78 & $\mathbf{1 . 5 1}$ & 1.66 \\
\hline
\end{tabular}

Nota: Quando $\alpha=2$ a distribuição é normal. Quando ele é menor do que 2, as caudas são gordas e há maior ocorrência de eventos extremos do que seria esperado para uma distribuição normal. A tabela está organizada em ordem alfabética, índices MSCI ao fim. Os valores em negrito indicam o menor alfa obtido. 


\section{Notas}

${ }^{1}$ Os autores agradecem à Datastream e à Economática o uso dos seus dados, além do apoio de pesquisa do CNPq, da FAPERJ e do Instituto COPPEAD de Administração.

${ }^{2}$ Como Fama (1965a, 1965b), Walter (1990, 1991), Zajdenweber (1994), Belkacem (1996) e Groslambert e Kassibrakis (1999) para ações e índices de ações e Cornew, Town e Crowson (1984), Walter (1994) e Corazza, Malliaris e Nardeli (1997) para vários mercados futuros.

\section{ReferênCias Bibliográficas}

AGGARWAL, R.;

LEAL, R. P. C.;

INCLÁN, C.

Volatility in emerging stock markets. Journal of Financial and Quantitative Analysis, v. 34, n. 1, p. 33-50, 1999.

BACHELIER, L.

Téorie de la spéculation. Annales de l'Écoles Normale Supérieure, p. 21-86, 1900.

\section{BEKAERT, G.}

Market integration and investment barriers in emerging markets. In: CLAESSENS, S.; GOOPTU, S. (Org.). Portfolio investments in developing countries. [S.l.], 1993. World Bank discussion paper 228, p. 221-251.

BELKACEM, L.

Processus stables et applications en finance. Paris, 1996. Thèse (Doctorat en Sciences Mathématiques) Université Paris IX Dauphine.
CORAZZA, M.;

MALLIARIS, A. G.;

NARDELLI, C.

Searching for fractal structures in agricultural futures markets. Journal of Futures Markets, v. 17, n. 4, p. 433-473, 1997.

CORNEW, R.;

TOWN, D.;

CROWSON, L.

Stable distribution, futures prices and the measurement of trading performance. Journal of Futures Markets, v. 4, p. 531557, 1984.

DUMOCHEL, W. H.

Stable distributions in statistical inference. [S.l.], 1971. Thesis (Ph.D.) Department of Statistics, Yale University.

FAMA, E. F.

The behavior of stock market prices. Journal of Business, $v$. 38, p. 34-105, 1965a. 
Portfolio analysis in a stable paretian market. Management Science, v. 11, p. 404-419, 1965b.

GOPIKRISHNAN, P. et al.

Scaling of the distribution of financial market indices. Physical Review, v. 60, n. 5, p. 5305-5316, 1999.

GROSLAMBERT, B.; KASSIBRAKIS, S.

The alpha-stable hypothesis: an alternative to the distribution of emerging market returns. Emerging Markets Quarterly, p. 21-38, Spring 1999.

\section{INTERNATIONAL FINANCE CORPORATION.}

Emerging stock markets fact book 1999. Washington, DC: IFC Publications, 1999.

LEAL, R. P. C.;

DA SILVA, A. L. C.; RIBEIRO, T. S.

Alocação ótima de ativos em fundos de pensão brasileiros. Relatório COPPEAD 351, 2001.

LÉVY, P.

Calcul des probabilités. Paris: 1925.

MANDELBROT, B. B.

The variation of certain speculative prices. Journal of Business, v. 36, p. 394-419, 1963.
MCCULLOCH, J. H.

Simple consistent estimators of stable distribution parameters. Commun. Statist. - Simula, v. 15, n. 4, p. 1109-1136, 1986.

MICHAEL, J. R.

The stabilized probability plot.

Biometrika, v. 70, p. 11-17, 1983.

MICHAUD, R.

Efficient asset management. Boston: Harvard Business School Press, 1998.

NOLAN, J. P.

Fitting data and assessing goodness-of-fit with stable distributions. Department of Mathematics and Statistics, American University, June 1999. mimeo.

OSBORNE, M. F. M.

Brownian motion in the stock market. Operations Research, v. 7, p. 145-173, 1959.

PETERS, E.

Fractal market analysis. New York: John Wiley \& Sons, 1994.

SAMORODNITSKY, G.;

TAQQU, M. S.

Stable non-gaussian random processes. New York: Chapman and Hall, 1994. 
TORRES, R.;

BONOMO, M.;

FERNANDES, C.

A aleatoriedade do passeio na Bovespa: testando a eficiência do mercado acionário brasileiro. Ensaios Econômicos da EPGE, 402, 2000.

WALTER, C.

Lévy-stable distributions and fractal structure on the Paris market. In: AFIR INTERNATIONAL COLLOQUIM, 3., Paris. Proceedings... Paris: [s.n.], 1990. p. 242-259.

L'utilization des lois Levy-stable en finance: une solution possible au problème posée par les discontinuités des trajectoires boursières. Bulletin de L'Institut des Actuaires Français, 329, p. 3-32, 1991.

Les structures des hasards en économie: efficience des marchés, lois stables et processus fractals. Paris, 1994. Thèse (Doctorat ès Science Economiques) - Institut d'Etudes Politiques.

ZAJDENWEBER, D.

Self-similar properties of the CAC40 Index. Revue d'Economie Politique, v. 104, p. 407-434, 1994.

ZOLOTAREV, V. M.

One-dimensional stable distributions. Amer. Math. Soc. Transl. of Math. Monographs, v. 65, 1986. 